Biology | Pedro M. Rodrigues \& Cláudia Raposo de Magalhães

\title{
New ways to assess stress in fish are urgently needed in aquaculture
}

\begin{abstract}
With an increasing demand in fish products, aquaculture is fast becoming a main priority towards achieving sustainable fish production. In this context, an accurate and consistent way is essential to ensure high standards of welfare Dr Pedro Miguel Rodrigues and Msc Cláudia Raposo de Magalhães, based at the University of Algarve, CCMAR, Portug believe current methods are unreliable and a new approach is urgently needed. bort option is to us, cuttingedge new technologies like metabolomics and proteomic to find markers which can be used to assess the level of stress in fish.
\end{abstract}

With an increasing demand in fish
roducts, aquaculture is fast becoming products,
a main pri

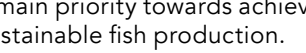

umans have explored bountiful have their own guidelines and practica indications on stocks to ensure their welfare. generations, fishermen deemed it to be infinitely vast, but it's becoming increasingly apparent that our waters are These guidelines, however, are based not as indestructible as we once believe he oceans cannot conthue to cover Responsible and sustainable fisher is crucial to move forward, and aquaculture may be the best tool to fill this gap.

However, aquaculture can have devastating effects on animal welfare The industry and the legislators have attempted to implement codes of practice to ensure companies take fish welfare seriously and promote good husbandry conditions. The Federation of European Aquaculture Producers, the World Organisation for Anima Health and even the European Union 列 biology. Dr Pedro Miguel Rodrigues nd Msc Claudia Raposo de Magalhães, based at the University of Algarve, Portugal, believe that, to develop appropriate welfare protocols, it is of each species.

A wide-ranging protocol doesn't suit the several species of fish used in aquaculture, each one with its own anatomical, physiological and behavioural characteristics. Instead, the researchers defend that the industry needs to develop ways to assess each species.

\section{CAN FISH FEEL HAPPY OR SAD?} \section{Up until now, the idea of fish welfare} has mostly been synonymous with merely keeping the animals alive and in good health. However, recent research is starting to unveil that these animals need more than that. It turns out that, despite lacking selfawareness and cognitive abilities on the same level as mammals, fish can nevertheless experience pain and fear and are even capable of expressing rudimentary emotions.

For Dr Rodrigues and Raposo de Magahanes, this research means that fish can adapt and evolve if the environment around them changes, and a stressful situation arises. In fact, "Goldilocks" approct ilsh have a to stress: too much will distress the animals, and they will struggle to cope, they'll get bored. On the other hand, an intermediate level of stress is just right trigger a sense of reward and teach the animals how to cope with future stressful situations.

For Dr Rodrigues and Raposo de Magalhães, welfare is not just a matter of looking at the animal's health but also includes what the fish is deeper understanding of the menta processes in fish and explore to what extent fish are able to make conscious expectations", said the researchers.

\section{ASSESSING STRESS IS NOT EASY}

This ability to adap

ment should really not be that surprising. In the wild, fish are exposed to a variety of situations which may cause stress. From injuries and parasites to predators and storms, fish need coping mechanisms to deal with such adverse conditions. The animals may be temporarily stressed, but that .

In contrast, what we consider harmless practices in aquaculture - such as repetitive handing or high fish cansity - can be severe stress factors

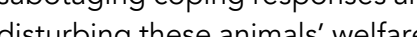

It's impossible to say whether a situation is stressful or not without fish. For Dr Rodrigues and Raposode Magalhães, this is paramount to ensure production. "Improving the quality of husbandry practices and the measurement of welfare to reduce

There is a need to establish new methods that will promote productivity and consumer's cceptance of farmed fish.

ress should not be seen only from a productive point of view but also from perspective", says Dr Rodrigues.$$
\text { "Thermal a few hours later, it's virtually }
$$

The problem now is finding an this. High levels of play to achieve are currently used as the prim indicator of stress in fish, but this has limited value Dr Rodrigues and Raposo de Magalhães believe this information is not reliable enough to$$
\text { check fish welfare. }
$$
This hormone can be influenced by many different factors, including the frequency of exposure and animal genetics. For example, although most fish show a pattern of increased contisol levels after exposure to a to normal a few hours later, it's virtually

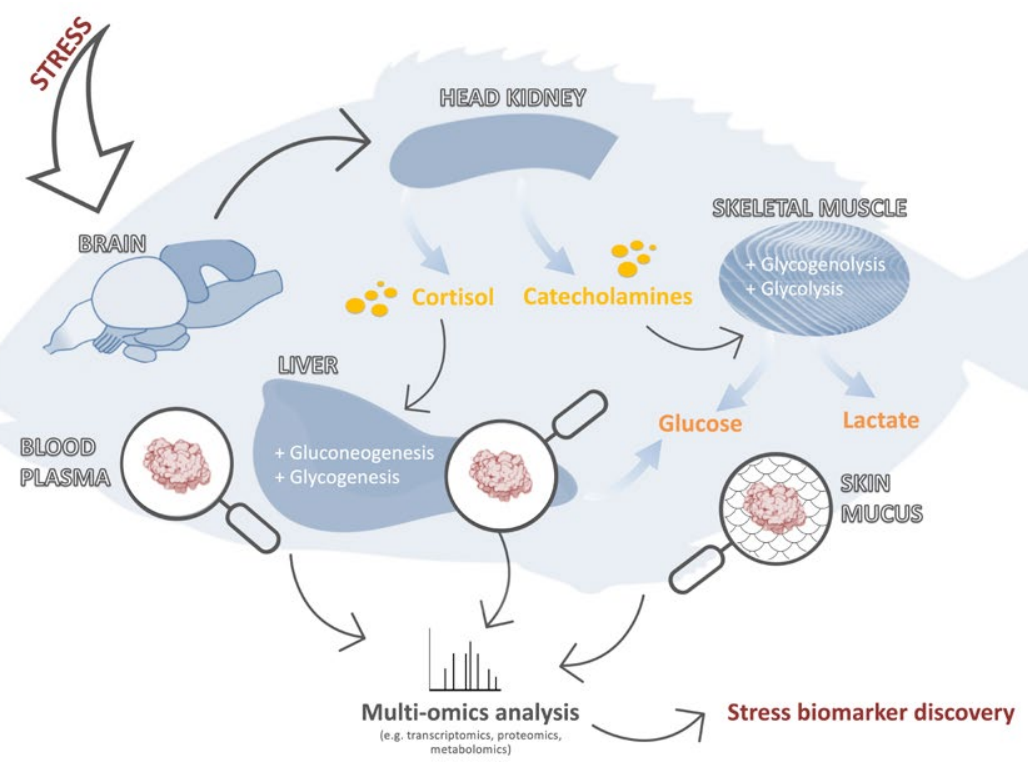

Integration of lifferent 'omics technologies can provide a hlistic oveniew of potential alterations in the
fish biological system under stressful situations, thus allowing the discovery of reliable stress indicators. 


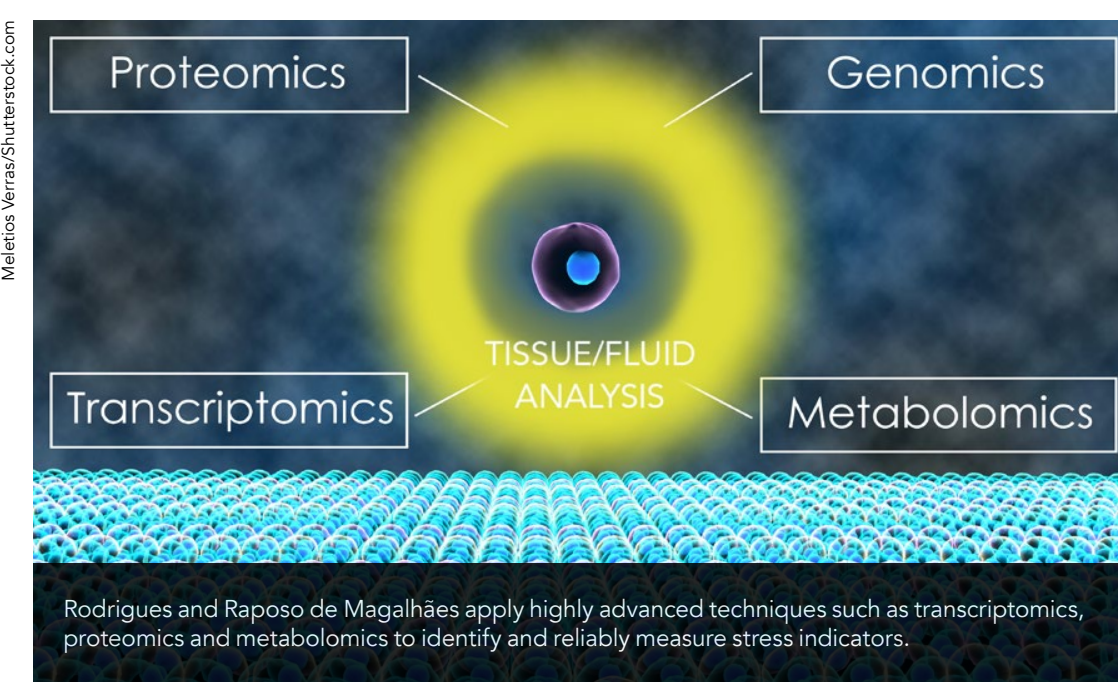

impossible to define what 'normal' is for each animal.

The reliability of cortisol levels subjected to prolonged and chre stress. For these animals, cortisol may stay elevated for days or even weeks or may return to low levels even though the animal remains stressed. It's impossible to predict how each anima reacts, but most likely the situation will lead to stunted growth, low performance, impaired reproductive
function and poor health, disease and ultimately death.

In theory, there are other compounds such as glucose and lactate - that ca reflect conditions of poor welfare. However, the same issues arise, and results must be interpreted with care, considering extinsic factors such as di season of the yincer, to nast feding and

Dr Rodrigues and Raposo de Magalhães defend none of thes compounds is a reliable indicator of stress due to their high biological. variability. "We believe these methods are unreliable", they explain, "there is a need to establish new methods that will promote productivity and consumer's acceptance of farmed fish".

\section{NEW OPTIONS TO}

\section{MONITOR STRESS}

an effort to find the Holy Grail of stress indicators, many scientists have started using highly advanced proteomics and metabolomics. In a just a few. There's still a long road Uust a few. There's sil a long roins, and good starting point.

For Dr Rodrigues and Raposo approaches are also likely to come into play in the future. A combined effort using transcriptomics, proteomics and metabolomics "provide a wider vision of the physiological function of interest while pointing out the direction for future research", said the researchers. "In the next years, with the development of more cost-effective technologies and the increase in proteomic databases, the biology research will be enhanced". e enhanced".

\section{FUTURE DIRECTIONS}

Consumers are getting increasingly issues. Keeping up with high welffer standards is probably the most critica factor in how consumers perceive the aquaculture industry.

incredible de Magalhães believe there is no reason why these procedures cannot applied to aquaculture as well. These This is where science comes in. Welfare is no longer a matter of

Identification of reliable biomarkers of fish welfare would greatly improve our knowledge regarding responses to stress. nutrition, reproduction and health but designing adequate husbandry also welfare and effects of stress.

Undoubtedly, proteomics has been the most common method used in aquaculture so far. Studies looking at the impact of diseases, water temperature, stocking densities, have uncovered exciting changes the type and abundance of specific proteins produced in stressed fish.

One of the main aims of this work is to find a few of these proteins that can reliably work as stress indicators. There are some good news in this front, as some potential markers have been identified. The list includes proteins involved in transporting iron, protecting against oxidative damage procedures to ensure fish are in good it has to provide an environment where fish can thrive and grow as if in the wild Fish can deal with a certain amount of stress in their lives, but how much is too much? Dr Rodrigues and Raposo de Magalhães believe in-depth knowledge of fish biology is the best pathway to finding the most effective welfare assessment tools. reliable biomarkers of fish welfare would greatly improve our knowledge regarding responses to stress" conclude the researchers and contribute to the development of more concern to a more holistic, ecological
In this context, "identification of sustainable fish rearing protocols with

\section{Behind the Research (f) ก \\ Cláudia Raposo de Magalhães}

E: pmrodrig@ualg.pt T: $+351-289800100$ (ext:7855) W: https://welfish.com/

Research Objectives

Pedro Rodrigues and Cláudia Raposo de Magalhães investigate reliable and standardised tests for farmed fish welfare assessment.

\section{Detail}

\section{Address}

Universidade do Algarve, Campus de

FCT, Ed. 7, 8005-139, Faro, Portugal

Bio

Both Pedro Rodrigues and Cláudia Raposo de Magalhães are members Research group at CCMAR, University

\section{References}

Raposo de Magalhães C, Cerqueira M, Schrama D, Moreira M, Boonanuntanasarn S, Rodrigues P. (2020) A Proteomics and other Omics approach in the context of farmed fish welfare and biomarker discovery. Reviews in Aquaculture, 12. 122-144.

\section{CCMAR | UAlg}

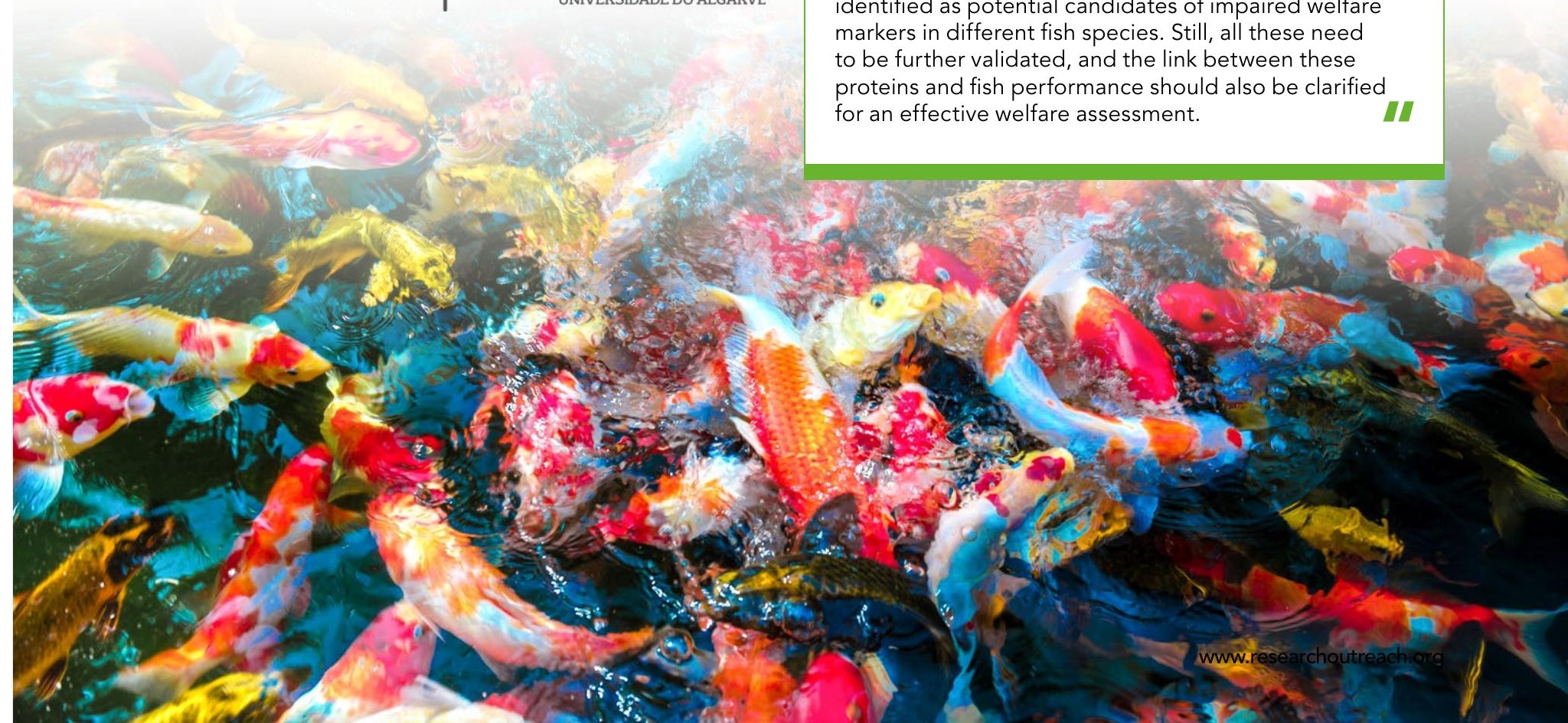

